\title{
Signe (ou syndrome) de Chilaiditi
}

\section{Chilaiditi's sign (or syndrome)}

\author{
S. Sidorkiewicz $\cdot$ V. Nouyrigat $\cdot$ G. Chéron \\ Reçu le 17 juillet 2013; accepté le 22 août 2013 \\ (C) SFMU et Springer-Verlag France 2013
}

Un enfant de 5 ans est examiné aux urgences pour anorexie, douleurs abdominales intermittentes évoluant depuis un an et stagnation pondérale. L'examen clinique abdominal est sans particularité. Le cliché d'abdomen sans préparation (ASP) retrouve la présence d'air (avec des haustrations coliques) entre le foie et la coupole diaphragmatique droite (Fig. 1). Une attitude conservatrice a été décidée et aucune autre imagerie n'a été effectuée. D'autres examens complémentaires ont été demandés pour poursuivre l'enquête étiologique des douleurs abdominales, avant de conclure à un lien éventuel entre les signes fonctionnels digestifs et le signe de Chilaiditi. Décrit en 1910 par un radiologue grec qui lui donna son nom, le signe de Chilaiditi correspond à l'interposition d'une partie du côlon entre le foie et la coupole diaphragmatique droite. Radiologiquement, l'hémidiaphragme droit est surélevé par rapport au foie, le côlon est distendu par l'air et la marge supérieure du foie est au dessous du niveau de l'hémidiaphragme gauche. Les haustrations coliques permettent le diagnostic différentiel avec un pneumopéritoine ou un abcès sous-phrénique [1]. Phénomène rare ( 0,025 à $0,28 \%$ de la population), il est plus fréquent chez les hommes, notamment âgés, souffrant de retard mental ou sous traitement psychotrope. Il est le plus souvent asymptomatique (signe de Chilaiditi) et découvert fortuitement ; il est parfois responsable (syndrome de Chilaiditi) de douleurs abdominales, constipation, vomissements, anorexie, détresse respiratoire, voire de complications telles que volvulus et occlusion. Des facteurs favorisants ont été décrits : absence ou laxité des ligaments suspenseurs ou du ligament falciforme, aérophagie, ascite, grossesses multiples, élévation de la coupole diaphragmatique droite (paralysie,

\footnotetext{
S. Sidorkiewicz $\cdot$ V. Nouyrigat $(\varangle) \cdot$ G. Chéron

Service des urgences pédiatriques,

Groupe hospitalier Necker-Enfants Malades,

Assistance publique-hôpitaux de Paris, 149, rue des Sèvres,

F-77743 Paris cedex 15, France

e-mail : valerie.nouyrigat@nck.aphp.fr

G. Chéron

Université Paris Descartes, 12, rue de l'école de médecine, F-75006 Paris, France
}

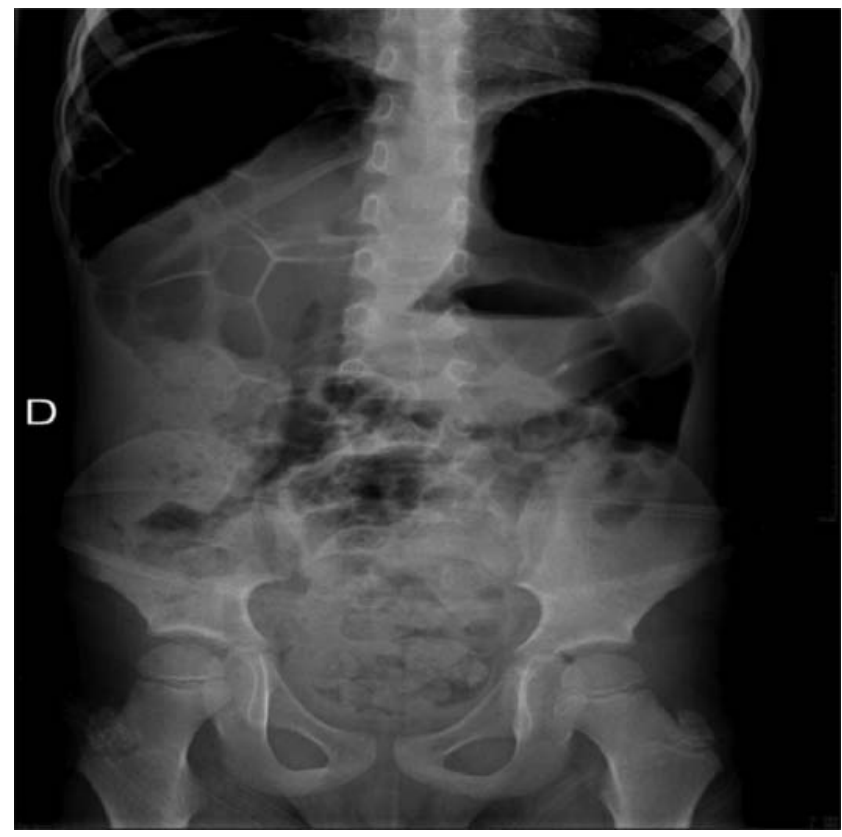

Fig. 1 Cliché d'abdomen sans préparation. Signe de Chilaiditi : interposition colique hépatodiaphragmatique

éventration), dolichocôlon, constipation chronique, alitement prolongé, malrotation intestinale, emphysème pulmonaire. La connaissance de ce signe radiologique est importante pour éviter des interventions chirurgicales inutiles. Le traitement est en effet le plus souvent conservateur. Aux urgences, la prise en charge initiale d'un patient symptomatique associe repos au lit, hydratation intraveineuse et traitement laxatif. Au décours, la disparition de l'air sous-diaphragmatique et le repositionnement de l'intestin en position normale peuvent être constatés sur un ASP, élément supplémentaire en faveur du diagnostic. Le traitement chirurgical (résection colique, colopexie) est réservé aux cas de volvulus, d'occlusion ou de perforation.

\section{Références}

1. Moaven O, Hodin RA (2012) Chilaiditi Syndrome, a rare entity with important differential diagnoses. Gastroenterol Hepatol 8:276-8 